\title{
No evidence for a diurnal vasoactive intestinal polypeptide (VIP) rhythm in the human suprachiasmatic nucleus
}

\author{
Michel A. Hofman ${ }^{*}$, Jiang-Ning Zhou, Dick F. Swaab \\ Netherlands Institute for Brain Research, Graduate School of Neurosciences, Meibergdreef 33, 1105 AZ Amsterdam, The Netherlands
}

Accepted 30 January 1996

\begin{abstract}
The mammalian suprachiasmatic nucleus $(\mathrm{SCN})$ is implicated in the temporal organization of circadian rhythms in a variety of physiological, endocrine and behavioral processes. Since the environmental light-dark cycle is the main zeitgeber for many of these rhythms, photic information may have a synchronizing effect on the endogenous clock of the $\mathrm{SCN}$ by inducing periodic changes in the activity of certain groups of neurons. The present study was performed to investigate the diurnal profile of the vasoactive intestinal polypeptide (VIP)-producing neurons in the SCN of humans. No significant diurnal variations were found in the volume of the VIP subdivision of the SCN nor in the number of VIP-producing neurons. In contrast with the VIP cell population, the subdivision of the human SCN containing vasopressin-producing neurons has previously been reported to exhibit a distinct diurnal rhythm, with low values during the night and peak values during the early morning. These findings suggest that the expression of vasopressin, but not that of VIP, in the human SCN exhibits an endogenous circadian rhythm.
\end{abstract}

Keywords: Suprachiasmatic nucleus; Human hypothalamus; Vasoactive intestinal polypeptide; Vasopressin; Circadian rhythm

\section{Introduction}

The suprachiasmatic nucleus ( $\mathrm{SCN}$ ), a group of parvocellular neurons located in the basal part of the anterior hypothalamus, is considered to be the principal component of the mammalian biological clock. It is responsible for generating and coordinating many physiological, endocrine and behavioral rhythms having a period of approximately $24 \mathrm{~h}$ (for reviews, see [22,29,33,37,46]).

The environmental light-dark cycle is the main Zeitgeber in most mammals, including humans. Therefore, photic information may have a synchronizing effect on the clock mechanism of the SCN by inducing changes in the functional activity of certain groups of neurons. As in other mammals, the human SCN contains distinct populations of chemically defined neurons (e.g. vasopressin (VP), somatostatin (SS), neuropeptide-Y (NPY), and vasoactive intestinal polypeptide (VIP)), some of which may be involved in the temporal organization of these circadian rhythms (see e.g. $[19,22,28,30]$ ). Animal studies have shown that the peptide content and mRNA levels of some

\footnotetext{
${ }^{*}$ Corresponding author. Fax: (31) (20) 696-1006.
}

of these SCN neuronal subpopulations, such as the VP-, NPY-, and SS-immunoreactive neurons, exhibit circadian rhythms, even when animals were kept in constant darkness $[13,21,38-40,44,50]$, indicating that the biosynthetic activity of these neurons is controlled by an intrinsic circadian pacemaker, independent of external lighting conditions. Other peptidergic cell groups in the SCN, such as the VIP-immunoreactive neurons, respond to photic stimuli, but do not show significant diurnal oscillations under constant dark conditions $[1,3,34,39,42,43]$.

VIP neurons are located in the ventrolateral subdivision of the SCN $[6,8,17,28,31,51]$, where photic and other information from various sources outside the SCN converges and is integrated with temporal information on the circadian pacemaker $[9,18,32]$. In fact, most afferent fibers to the SCN, including the retinohypothalamic tract (RHT) and geniculo-hypothalamic tract (GHT) project preferentially to this part of the $\operatorname{SCN}[12,18,20,41]$, where they make synaptic contacts with VIP neurons. Therefore, the VIP subdivision of the SCN could be regarded as part of the timekeeping mechanism that mainly deals with light response or entrainment to environmental lighting conditions. The present investigation was performed to determine whether the daily light-dark cycle affects the func- 
tional activity of VIP-expressing neurons in the human SCN.

\section{Materials and methods}

Brain tissue of 36 human subjects (18 males and 18 females), ranging in age from 6 to 92 years, was obtained at autopsy. Brains were collected and neuropathologically examined either in the Free University Hospital (Prof. dr. F.C. Stam, Dr. W. Kamphorst) or in the Academic Medical Center (Dr. D. Troost), both in Amsterdam, the Netherlands, between 1981 and 1993. Patients with a primary neurological or psychiatric disorder were excluded. Brains were removed from their skulls generally within $48 \mathrm{~h}$ postmortem, weighed, and immediately put in $4 \%$ formaldehyde at room temperature for about 1 to 2 months. The hypothalamic area containing the $\mathrm{SCN}$ was dissected from each brain, dehydrated and embedded in paraffin. Serial 6 $\mu \mathrm{m}$ frontal sections were cut at a microtome, mounted on chrome-aluminum-sulphate-coated slides, deparaffinized, hydrated and brought to Tris-buffered saline. The cytoarchitectonic boundaries of the SCN, which are indistinct in thin sections if conventional stains are applied (see e.g. $[17,28]$ ), were delineated by using an antiserum against VIP as a marker, and every 25 th section was stained (for details on the immunocytochemistry, see [51]).

Cross-sectional area measurements through the SCN were performed unilaterally. The volume of the VIP cell population in the SCN was determined by integrating all sectional areas that contained immunocytochemically stained cells, taking into account section thickness and the magnification at which the original tracings were made [35]. The number of VIP-immunoreactive neurons in the SCN of each subject was estimated by counting the number of nuclear profiles per unit area in immunocytochemically stained material, followed by a discrete deconvolution procedure [47], which included a modification proposed by Cruz-Orive [11] and a correction for section thickness (for further details see $[14,17]$ ). The number of VIP-expressing neurons in the SCN was compared with that of the VP cell population, which in $89 \%$ of the cases was done by measuring these peptidergic neurons in adja- cent sections in the same SCN. Subjects were grouped into four diurnal periods based on the time of death (see Table 1). Since a number of aspects of the circadian timing system already begin to change substantially in the fifth decade of life $[16,36,45]$ and the number of VIP-expressing neurons in the SCN of men already starts to decline between 40 and 50 years [17,51], the cohort was divided into two age groups: 'young subjects', who were under 40 years of age $(26.0 \pm 2.4$ years, $n=18)$ and 'elderly subjects', who were older than 40 years $(66.6 \pm 4.4$ years, $n=18$ ). Differences among the diurnal groups were statistically evaluated by the non-parametric Kruskal-Wallis test (two-tailed) because of non-normality of the distributions and heteroscedasticity [10]. A two-way analysis of variance (ANOVA) was used to test for the combined effects of time of day and sex. Throughout this study values are expressed as mean \pm S.E.M.

\section{Results}

The subdivision of the human SCN containing VIP-expressing neurons did not show any significant diurnal variations, neither in volume $(P>0.4)$, nor in VIP cell number $(P>0.5)$ (Table 1$)$. In fact, no significant diurnal fluctuations in any of the VIP parameters measured, including the VIP neuronal density, could be detected. It should be noted that the high VIP values observed at dusk, which, however, are not significantly different from those observed at other periods, are mainly due to the disproportionate contribution of young males to this diurnal group. As is known from previous studies, the SCN of especially these subjects contain large numbers of VIP-producing neurons [17,51]. In contrast with the VIP cell population, the VP-expressing neurons in the human SCN have been shown to exhibit a marked diurnal pattern [15,16]. These diurnal oscillations were, however, only found in young, but not in elderly subjects. Since age may be a confounding factor, masking the possible photic effects of the daily light-dark cycle on the VIP synthesizing activity, a further analysis was performed in which young and elderly people were distinguished. Again, no significant diurnal variations in the VIP subdivision of the SCN could be detected in

Table 1

Diurnal variations in the vasoactive intestinal polypeptide (VIP) neuronal subdivision of the human suprachiasmatic nucleus (SCN)

\begin{tabular}{llrlll}
\hline Time of day & Period $(\mathrm{h})$ & No. of subjects & \multicolumn{2}{l}{ Suprachiasmatic nucleus ${ }^{\mathrm{a}}$} \\
\cline { 3 - 5 } & & & VIP volume $\left(\mathrm{mm}^{3}\right)$ & VIP cell number $\left(\times 10^{3}\right)$ & VIP cell nuclear diameter $(\mu \mathrm{m})$ \\
\hline Dawn & $06.00-10.00$ & 8 & $0.0243 \pm 0.0046$ & $1.36 \pm 0.15$ & $7.02 \pm 0.37$ \\
Daytime & $10.00-18.00$ & 13 & $0.0362 \pm 0.0086$ & $1.65 \pm 0.22$ & $6.50 \pm 0.36$ \\
Dusk & $18.00-22.00$ & 5 & $0.0529 \pm 0.0149$ & $2.21 \pm 0.64$ & $6.35 \pm 0.43$ \\
Nighttime & $22.00-06.00$ & 10 & $0.0359 \pm 0.0077$ & $1.89 \pm 0.28$ & $6.60 \pm 0.15$ \\
Statistics ${ }^{b}$ & & & $P=0.477$ & $P=0.511$ & $P=0.454$ \\
\hline
\end{tabular}

\footnotetext{
${ }^{\text {a }}$ Values are given as mean \pm S.E.M.

${ }^{\mathrm{b}}$ Kruskal-Wallis multiple comparisons test.
} 


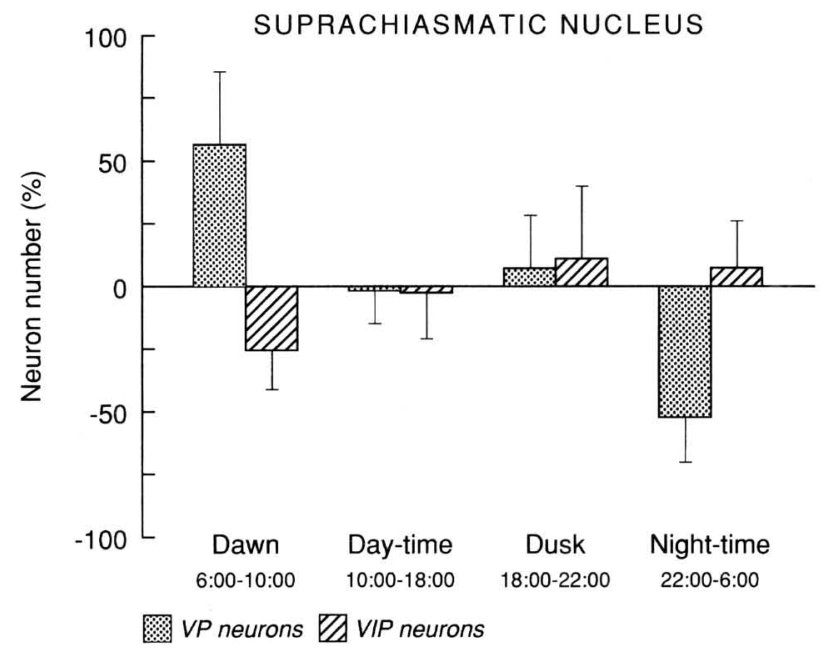

Fig. 1. Diurnal fluctuations in the number of vasopressin (VP)- and vasoactive intestinal polypeptide (VIP)-expressing neurons in the human suprachiasmatic nucleus (SCN). Subjects were grouped into four periods based on the time of death (see Table 1). Data are presented as deviations from the weighted 24-h mean ( \pm S.E.M.). Significant diurnal fluctuations were only observed in the group of VP-expressing neurons $(P=0.028)$.

either age group ( $P>0.15$ in both groups). In young subjects, the nighttime number of VIP-expressing neurons in the SCN was similar to that during the day $(2.13 \pm 0.40$ $\times 10^{3}$ and $1.93 \pm 0.35 \times 10^{3}$, respectively; $P=0.898$ ) (Fig. 1). Furthermore, there was no interaction between time of day and $\operatorname{sex}(F(3,10)=1.90 ; P=0.194)$. We therefore conclude that the time of day does not have any effect on the number of VIP-expressing neurons in the human SCN and that the sexes do not differ in this respect.

The VP subdivision of the SCN of young subjects, on the other hand, exhibits a marked diurnal oscillation, containing more VP-expressing neurons during the daytime than during the nighttime $\left(7.60 \pm 1.01 \times 10^{3}\right.$ and $4.13 \pm$ $1.15 \times 10^{3}$, respectively; $P=0.028$ ) (see Fig. 1). It should be noted that these diurnal variations in the VP subdivision cannot be explained by differences in age, postmortem delay or fixation time, since no statistically significant differences were found among the diurnal periods examined for any of these parameters ( $P>0.2$ in all cases).

\section{Discussion}

As the number of VIP-immunoreactive neurons probably reflects the peptidergic activity state of the cells, these results suggest that the synthesis of VIP in the human SCN does not exhibit diurnal fluctuations, at least not under semi-natural lighting conditions. Though VIP neurons in the SCN are sometimes thought to be implicated in the rhythm generation of the circadian clock $[6,25,26]$, there is no direct indication that they really are pacemaker neurons. However, observations in the SCN of rodents have shown that concentrations of VIP immunoreactivity and VIP
mRNA oscillate with a period of $24 \mathrm{~h}$ under light-dark conditions $[2,34,43,52]$, although this oscillation may be driven by a photic stimulus $[33,39]$. An argument in favour of this hypothesis is that VIP levels were found to be stable throughout the diurnal cycle in animals that were kept in constant darkness or were exposed to constant light. These observations in fact suggest that the VIP synthesis in the SCN is characterized by the absence of an endogenous rhythm and that the VIP level reflects the external lighting conditions and could thus mediate photic information to the pacemaker.

It appears that light intensity is an important parameter in synchronizing circadian rhythms to the environmental light-dark cycle (see e.g., $[5,23,48])$. Although low light intensities $(<500$ lux $)$ have been reported to have a synchronizing effect on the SCN [5,24], other studies have demonstrated that higher light intensities ( $>1000$ lux) are required to exert a direct biological effect on the human circadian pacemaker $[4,7,27,49]$. Therefore, the absence of a distinct diurnal rhythm in the VIP-expressing neurons in the human SCN, as found in the present study, might be due to the artificial lighting conditions to which most terminal patients are exposed at the end of their lives. This also explains why the VP-expressing neurons in the human SCN exhibit diurnal fluctuations $[15,16]$, even when the lighting conditions are inadequate to induce diurnal oscillations in synthesis rate at the input side of the SCN, where VIP and other RHT recipient neurons are located. It appears, namely, that the VP content in the SCN of rodents exhibits circadian rhythms under LD and DD conditions (see [19]). Persistence of VP levels under constant conditions suggest that VP neurons, in contrast to VIP neurons, oscillate endogenously, independent of the lighting conditions. However, it does not necessarily mean that VP neurons are pacemaker cells. The role of VP in the circadian timing system may be limited to being an output mediator linking the pacemaker and the effector that acts outside the circadian clock. These studies support the idea that not all neurons in the SCN are pacemaker neurons and that VIP-expressing neurons may have to be excluded from this function.

\section{Acknowledgements}

The authors are grateful to Mr. B. Fisser for technical assistance, Mr. H. Stoffels for the graphical work and Ms. W.T.P. Verweij for secretarial assistance. Human brain tissue was obtained from the Netherlands Brain Bank in Amsterdam (coordinator Dr. R. Ravid).

\section{References}

[1] Albers, H.E., Minamitani, N., Stopa, E. and Ferris, C.F., Light selectively alters vasoactive intestinal peptide and peptide histidine 
isoleucine immunoreactivity within the rat suprachiasmatic nucleus, Brain Res., 437 (1987) 189-192.

[2] Albers, H.E., Stopa, E.G., Zoeller, R.T., Kauer, J.S., King, J.C., Fink, J.S., Mobtaker, H. and Wolfe, H., Day-night variation in prepro vasoactive intestinal peptide/peptide histidine isoleucine mRNA within the rat suprachiasmatic nucleus, Mol. Brain Res., 7 (1990) 85-89.

[3] Albers, H.E., Liou, S.-Y., Stopa, E.G., and Zoeller, R.T., Interaction of colocalized neuropeptides: functional significance in the circadian timing system, J. Neurosci., 11 (1991) 846-851.

[4] Boivin, D.B., Duffy, J.F., Kronauer, R.E., and Czeisler, C.A., Sensitivity of the human circadian pacemaker to moderately bright light, J. Biol. Rhythms, 9 (1994) 315-331.

[5] Bojkowski, C.J., Aldhous, M.E., English, J., Franey, C., Poulton, A.L., Skene, D.J. and Arendt, J., Suppression of nocturnal plasma melatonin and 6-sulphatoxy melatonin by bright and dim light in man, Horm. Metabol. Res., 19 (1987) 437-440.

[6] Botchkina, G.I. and Morin, L.P., Ontogeny of radial glia, astrocytes and vasoactive intestinal peptide immunoreactive neurons in hamster suprachiasmatic nucleus, Dev. Brain Res., 86 (1995) 48-56.

[7] Brainard, G.C., Lewy, A.J., Menaker, M., Fredrickson, R.H., Miller, L.S., Weleber, R.G., Cassone, V. and Hudson, D., Dose-response relationship between light irradiance and the suppression of plasma melatonin in human volunteers, Brain Res., 454 (1988) 212-218.

[8] Card, J.P., Brecha, N., Karten, H.J. and Moore, R.Y., Immunocytochemical localization of vasoactive intestinal polypeptide containing cells and processes in the suprachiasmatic nucleus of the rat: light and electron microscopic analysis, J. Neurosci., 1 (1981) 1289-1303.

[9] Card, J.P. and Moore, R.Y., Organization of lateral geniculate-hypothalamic connections in the rat. J. Comp. Neurol., 284 (1989) $135-147$.

[10] Conover, W.J., Practical Nonparametric Statistics, Wiley, New York, 1980.

[11] Cruz-Orive, L.M., Distribution-free estimation of sphere size distributions from slabs showing overprojection and truncation, with a review of previous methods, J. Microsc., 131 (1983) 265-290.

[12] François-Bellan, A.-M. and Bosler, O., Convergent serotonin and GABA innervation of VIP neurons in the suprachiasmatic nucleus demonstrated by triple labeling in the rat, Brain Res., 595 (1992) 149-153.

[13] Fukuhara, C., Shinohara, K., Tominaga, K., Otori, Y. and Inouye, S.-I.T., Endogenous circadian rhythmicity of somatostatin-like immunoreactivity in the rat suprachiasmatic nucleus, Brain Res., 606 (1993) 28-35

[14] Hofman, M.A., Fliers, E., Goudsmit, E. and Swaab, D.F., Morphometric analysis of the suprachiasmatic and paraventricular nuclei in the human brain: sex differences and age-dependent changes, $J$. Anat., 160 (1988) 127-143.

[15] Hofman, M.A. and Swaab, D.F., Diurnal and seasonal rhythms of neuronal activity in the suprachiasmatic nucleus of humans, J. Biol. Rhythms, 8 (1993) 283-295.

[16] Hofman, M.A. and Swaab, D.F., Alterations in circadian rhythmicity of the vasopressin-producing neurons of the human suprachiasmatic nucleus (SCN) with aging, Brain Res., 651 (1994) 134-142.

[17] Hofman, M.A., Zhou, J.-N. and Swaab, D.F., Suprachiasmatic nucleus of the human brain: An immunocytochemical and morphometric analysis, Anat. Rec., 244 (1996) 552-562.

[18] Ibata, Y., Takahashi, Y., Okamura, H., Kawakami, F., Terubayashi, H., Kubo, T. and Yanaihara, N., Vasoactive intestinal peptide (VIP)-like immunoreactive neurons located in the rat suprachiasmatic nucleus receive a direct retinal projection, Neurosci. Lett., 97 (1989) $1-5$

[19] Inouye, S.-I.T. and Shibata, S., Neurochemical organization of circadian rhythm in the suprachiasmatic nucleus, Neurosci. Res., 20 (1994) 109-130.

[20] Johnson, R.F., Morin, L.P. and Moore, R.Y., Retino-hypothalamic projections in the hamster and rat demonstrated using cholera toxin Brain Res. 462 (1988) 301-312.

[21] Kalsbeek, A., Buijs, R.M., Engelmann, M., Wotjak, C.T. and Landgraf, R., In vivo measurement of a diurnal variation in vasopressin release in the rat suprachiasmatic nucleus, Brain Res., 682 (1995) $75-82$.

[22] Klein, D.C., Moore, R.Y. and Reppert, S.M. (Eds.), Suprachiasmatic Nucleus; The Mind's Clock, Oxford University Press, New York, 1991.

[23] Kronauer, R.E. and Czeisler, C.A., Understanding the use of light to control the circadian pacemaker in humans. In L. Wetterberg (Ed.), Light and Biological Rhythms in Man, Pergamon, Oxford, pp. 217-236.

[24] Laakso, M.-L., Hätönen, T., Sternberg, D., Alila, A. and Smith, S., One-hour exposure to moderate illuminance (500 lux) shifts the human melatonin rhythm, J. Pineal Res., 15 (1993) 21-26.

[25] Laemle, L.K., Ottenweller, J.E. and Fugaro, C., Diurnal variations in vasoactive intestinal polypeptide-like immunoreactivity in the suprachiasmatic nucleus of congenitally anophthalmic mice, Brain Res., 688 (1995) 203-208.

[26] Lehman, M.N., Silver, R., Gladstone, W.R., Kahn, R.M., Gibson, M. and Bittman, E.L., Circadian rhythmicity restored by neural transplant. Immunocytochemical characterization of the graft and its integration with the host brain, J. Neurosci., 7 (1987) 1626-1638.

[27] Lewy, A.J., Wehr, T.A., Goodwin, F.K., Newsome, D.A. and Markey, S.P., Light suppresses melatonin secretion in humans, Science, 210 (1980), 1267-1269.

[28] Mai, J.K., Kedziora, O., Teckhaus, L. and Sofroniew, M.V., Evidence for subdivisions in the human suprachiasmatic nucleus, $J$. Comp. Neurol., 305 (1991) 508-525.

[29] Meijer, J.H. and Rietveld, W.J., Neurophysiology of the suprachiasmatic circadian pacemaker in rodents, Physiol. Rev., 69 (1989) 671-707.

[30] Moore, R.Y., The organization of the human circadian timing system, Prog. Brain Res., 93 (1992) 101-117.

[31] Moore, R.Y., Organization of the primate circadian system, J. Biol. Rhythms, 8 (suppl.) (1993) 3-9.

[32] Moore, R.Y. and Card, J.P., Intergeniculate leaflet: an anatomically and functionally distinct subdivision of the lateral geniculate complex, J. Comp. Neurol., 344 (1994) 403-430.

[33] Morin, L.P, The circadian visual system, Brain Res. Rev., 67 (1994) $102-127$.

[34] Morin, A., Denoroy, L. and Jouvet, M., Daily variations in concentration of vasoactive intestinal polypeptide immunoreactivity in hypothalamic nuclei of rats rendered diurnal by restricted feeding, Neurosci. Lett., 152 (1991) 121-124.

[35] Pakkenberg, B. and Gundersen, H.J.G., Total number of neurons and glial cells in human brain nuclei estimated by the disector and the fractionator, J. Microsc., 150 (1988) 1-20.

[36] Richardson, G.S., Circadian rhythms and aging. In C.E. Finch and E.L. Schneider (Eds.), Handbook of the Biology of Aging, Van Nostrand Reinhold, New York, 1990, pp. 275-305.

[37] Rusak, B., The mammalian circadian system: models and physiology, J. Biol. Rhythms, 4 (1989) 121-134.

[38] Shinohara, K., Isobe, Y., Takeuchi, J. and Inouye, S.-I.T., Circadian rhythms of somatostatin-immunoreactivity in the suprachiasmatic nucleus of the rat, Neurosci. Lett., 129 (1991) 59-62.

[39] Shinohara, K., Tominaga, K., Isobe, Y. and Inouye, S.-I.T., Photic regulation of peptides located in the ventrolateral subdivision of the suprachiasmatic nucleus of the rat: daily variations of vasoactive intestinal polypeptide, gastrin-releasing peptide and neuropeptide-Y, J. Neurosci., 13 (1993) 793-800.

[40] Södersten, P., De Vries, G.J., Buijs, R.M. and Melin, P., A daily rhythm in behavioral vasopressin sensitivity and brain vasopressin concentrations, Neurosci. Lett., 58 (1985) 37-41.

[41] Stopa, E.G., King, J.C., Lydic, R. and Schoene, W.C., Human brain 
contains vasopressin and vasoactive intestinal polypeptide neuronal subpopulations in the suprachiasmatic region, Brain Res., 297 (1984) $159-163$

[42] Takeuchi, J., Nagasaki, H., Shinohara, K. and Inouye, S.-I.T., A circadian rhythm of somatostatin messenger RNA levels, but not of vasoactive intestinal polypeptide/peptide histidine isoleucine messenger RNA levels in rat suprachiasmatic nucleus, Mol. Cell Neurosci., 3 (1992) 29-35.

[43] Takahashi, Y., Okamura, H., Yanaihara, N., Hamada, S., Fujita, S. and Ibata, Y., Vasoactive intestinal peptide immunoreactive neurons in the rat suprachiasmatic nucleus demonstrate diurnal variation, Brain Res., 497 (1989) 374-377.

[44] Tominaga, K., Shinohara, K., Otori, Y., Fukuhara, C. and Inouye, S.-I.T., Circadian rhythms of vasopressin content in the suprachiasmatic nucleus of the rat, NeuroReport, 3 (1992) 809-812.

[45] Touitou, Y. and Haus, E., Biological rhythms and aging. In Y. Touitou and E. Haus (Eds.), Biological Rhythms in Clinical and Laboratory Medicine, Springer, Berlin, 1992, pp. 188-207.

[46] Turek, F.W. and E. Van Cauter, Rhythms in reproduction. In E. Knobil and J.D. Neill (Eds.), Physiology of Reproduction, Raven, New York, 1994, pp. 487-540.
[47] Weibel, E.R., Stereological Methods, vol. 1, Practical Methods for Biological Morphometry, Academic Press, New York, 1979.

[48] Wetterberg, L., Melatonin in humans: Physiological and clinical studies, J. Neural. Transm., suppl. 13 (1978) 289-310.

[49] Wever, R.A., Einflu $\beta$ exogener Zeitgeber auf die zirkadiane Rhythmik, In J. Zulley, E. Haen, R. Lund and T. Roenneberg (Eds.), Biologische Rhythmen, S. Roderer Verlag, Regensburg, 1994, pp. 105-120.

[50] Yamase, K., Takahashi, S., Nomura, K., Haruta, K. and Kawashima, $\mathrm{S}$., Circadian changes in arginine vasopressin level in the suprachiasmatic nuclei in the rat, Neurosci. Lett., 130 (1991) 255-258.

[51] Zhou, J.-N., Hofman, M.A. and Swaab, D.F., VIP neurons in the human SCN in relation to sex, age, and Alzheimer's disease, Neurobiol. Aging, 16 (1995) 571-576.

[52] Zoeller, R.T., Broyles, B., Earley, J., Anderson, E.R. and Albers, H.E., Cellular levels of messenger ribonucleic acids encoding vasoactive intestinal peptide and gastrin-releasing peptide in neurons of the suprachiasmatic nucleus exhibit distinct 24-hour rhythms, $J$. Neuroendocrinol., 4 (1992) 119-124. 